\title{
Die Bautätigkeit der Päpste Julius II., Leo X. und Paul III. in Rom und deren Wahrnehmung in der ersten Hälfte des 16. Jahrhunderts
}

\author{
Thekla Kollmann \\ Kerngebiet: Neuzeit \\ eingereicht bei: Univ.-Ass. MMag. Markus Debertol \\ eingereicht im: SoSe 2020 \\ Rubrik: Proseminar-Arbeit
}

\begin{abstract}
Building Operations under Pope Julius II, Leo X and Paul III in Rome and their Perception in the First Half of the $16^{\text {th }}$ Century

The following seminar paper examines the perception of building projects in Rome under Pope Julius II, Leo X and Paul III in the first half of the $16^{\text {th }}$ century. On the one hand, contemporary reports show that measures to beautify and improve the streets of Rome generally left a positive impression among the Roman population. On the other hand, a letter written by Raffael Sanzio to Leo X criticized reconstruction efforts as they led to drastic changes, for example in demolishing numerous ancient monuments.
\end{abstract}

\section{Einleitung}

„Wie viele Päpste, Heiliger Vater, [...] haben antike Tempel, Statuen, Bogen und andere herrliche Gebäude zu Grunde gerichtet! [...] Das neue Rom, das wir jetzt erblicken, in seiner ganzen Größe und Schönheit, mit Palästen, Kirchen und andern Bauwerken geschmückt, ist, um es frei herauszusagen, durchaus mit Kalk gebaut, der aus antikem Marmor gewonnen wurde."1

\footnotetext{
1 Alfred von Reumont, Geschichte der Stadt Rom, Bd. 3, Abt. 2, Berlin 1870, S. 358. Originalzitat: „Quanti pontifici, Padre Santissimo, [...] hanno atteso a ruinare templi antiqui, statue, archi et altri ædificii gloriosi! [...] che ardirei dire che tutta questa Roma nova che hor si vede, quanto grande ch'ella si sia, quanto bella, quanto ornata di pallaggi, chiese et altri ædificii, tutta è fabricata di calce di marmi antichi!", zit. in Francesco Paolo di Teodoro, Lettera a Leone X di Raffaello e Baldassarre Castiglione (Biblioteca dell'“Archivum Romanicum”), Florenz 2020, S. 44.
} 
Mit diesen Worten kritisierte Raffael Sanzio die Zerstörung römischer Altertümer zur Gewinnung von Material für neue Bauwerke in einem Brief an Papst Leo X. ${ }^{2}$ Die Päpste der ersten Hälfte des 16. Jahrhunderts ließen Rom zu einer Renaissancestadt ausbauen. Durch die Errichtung von Palästen, Kirchen und Straßen erfuhr die Stadt eine Neugestaltung. ${ }^{3}$ Dabei traten die städtebaulichen Maßnahmen von Julius II., Leo X. und Paul III. hervor. Papst Julius II. (1503-1513) ${ }^{4}$ hatte neben vielen anderen Unternehmungen den Neubau der Peterskirche in Auftrag gegeben, welcher von seinen Nachfolgern fortgesetzt wurde..$^{5}$ Leo X. (1513-1521)6 förderte, wie sein Vorgänger, die städtische Bautätigkeit und trug zur Verschönerung der Straßen Roms bei.7 Paul III. (1534-1549)8 widmete sich, nach einer unruhigen Zeit, der Neugestaltung des Kapitols, dem Bau einer Triumphstraße anlässlich des Besuchs Kaiser Karls V. und der Fortsetzung des Neubaus der Peterskirche. ${ }^{9}$

Über die Bautätigkeit der genannten Päpste und besonders den Bau des Petersdoms sind zahlreiche Arbeiten erschienen, darunter etwa jene zur Architekturgeschichte Roms von Hubertus Günther ${ }^{10}$ und Marcello Fagiolo. ${ }^{11}$ Eine Publikation, die sich mit der zeitgenössischen Perzeption der Umgestaltung Roms auseinandersetzt, gibt es bislang noch nicht. Dennoch verwiesen Horst Bredekamp ${ }^{12}$ und Guido Rebecchini ${ }^{13}$ in ihren Arbeiten über den Neubau Sankt Peters und die Bautätigkeit Pauls III. auf zeitgenössische Berichte. Besonders relevant ist hier das Werk "Geschichte der Päpste seit dem Ausgang des Mittelalters" von Ludwig von Pastor, welches auf Quellen aus dem Vatikanischen Apostolischen Archiv basiert und dabei auf Berichte von Botschaftern, Klerikern, Humanisten und Literaten verweist.

Die eingangs zitierten Worte Sanzios deuten darauf hin, dass die Bautätigkeit der Päpste nicht nur positiv wahrgenommen wurde. Davon ausgehend wird in der vorliegenden Arbeit der Frage nachgegangen, inwiefern die städtebaulichen Maßnahmen unter den Päpsten Julius II., Leo X. und Paul III. in zeitgenössischen Quellen gelobt sowie kritisiert

2 Ludwig von Pastor, Geschichte der Päpste seit dem Ausgang des Mittelalters, Bd. 4: Geschichte der Päpste im Zeitalter der Renaissance und der Glaubensspaltung von der Wahl Leos X. bis zum Tode Klemens' VII. (1513-1534), Abt. 1: Leo X., Freiburg im Breisgau 1956, S. 467.

3 Loren Partridge, Renaissance in Rom. Die Kunst der Päpste und Kardinäle, Köln 1996, S. 14-15.

$4 \quad$ Ludwig von Pastor, Geschichte der Päpste, Bd. 3: Geschichte der Päpste im Zeitalter der Renaissance von der Wahl Innozenz' VIII. bis zum Tode Julius'II. 1484-1513, Abt. 2: Pius III. und Julius II., Freiburg im Breisgau 1956, S. 5.

5 Ebd., S. 915.

6 Pastor, Geschichte der Päpste, Bd. 4, S. 9.

7 Ebd., S. 387.

8 Ludwig von Pastor, Geschichte der Päpste, Bd. 5: Geschichte Papst Pauls III., Freiburg im Breisgau 1956, S. 9.

9 Kunibert Bering, Die Peterskirche in Rom. Architektur und Baupropaganda (Architektur der Welt), Weimar 2003, S. 38.

10 Hubertus Günther, L'urbanistica romana sotto il pontificato dei Medici, in: Henry A. Millon (Hrsg.), Rinascimento da Brunelleschi a Michelangelo. La rappresentazione dell'architettura, Mailand 1994, S. 546-551, https://doi. org/10.11588/artdok.00001273, eingesehen 8.8.2020.

11 Marcello Fagiolo dell'Arco, Das römische Pontifikat. Die Geschichte einer Ideologie im Spiegel einer heiligen Stadt, in: Maurizio Fagiolo dell'Arco (Hrsg.), Petersdom und Vatikan. Eine Dokumentation über das Zusammenwirken von Päpsten, Malern und Bildhauern, die Petersdom und Vatikan zu einem Wahrzeichen der Christenheit und Denkmal der Kunst machten, Freiburg im Breisgau 1983, S. 12-27.

12 Horst Bredekamp, Sankt Peter in Rom und das Prinzip der produktiven Zerstörung. Bau und Abbau von Bramante bis Bernini, Berlin 2000.

13 Guido Rebecchini, After the Medici. The New Rome of Pope Paul III., in: / Tatti Studies in the Italian Renaissance 11 (2007), Heft 1, https://www.jstor.org/stable/20111825, eingesehen 8.8.2020. 
wurden und worauf diese Reaktionen zurückzuführen sind. Basierend auf der Aussage Sanzios über die Zerstörung von Altertümern zu Gunsten prachtvoller Bauten wird die These aufgestellt, dass die Bautätigkeit in zeitgenössischen Quellen durchwegs negativ beurteilt wurde. Zur Beantwortung der Forschungsfrage wird im ersten Teil der Arbeit auf die positiven und im zweiten Teil auf die negativen Urteile über die päpstlichen Bautätigkeiten eingegangen, wobei die Urheber jener Kritiken sowie deren Perspektive genauer beleuchtet werden.

\section{Positive Beurteilungen der Bautätigkeit Papst Julius II., Leos X. und Pauls III.}

\subsection{Papst Julius II. als Mäzen der Künste}

Ein Zeugnis der Bewunderung der Bautätigkeit von Julius II. ist das Büchlein „Opuscu-

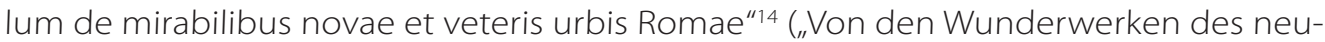
en und alten Rom") vom florentinischen Kanonikus Francesco Albertini. Er widmete das am 3. Februar 1510 erschienene Werk Julius II., welcher neben den Päpsten Nikolaus V. und Sixtus IV. das "neue Rom" gekennzeichnet habe. Der Autor rühmte Julius II. dafür, dass er beim Ausbau der Stadt, welchen Sixtus IV. begonnen hatte, all seine Vorgänger übertroffen habe. ${ }^{15}$ Wie kam Albertini zu diesem Urteil?

Zum einen muss angemerkt werden, dass Papst Julius II., wie bereits Nikolaus V. und Sixtus IV. vor ihm, das Ziel hatte, die geistliche Weltherrschaft der Kirche zu stärken, indem er Rom zu einem Zentrum der Kunst machen wollte. ${ }^{16}$ In der Baukunst knüpfte Julius II. durch die Anpassung der Verkehrswege Roms an den großen Pilgerstrom an seine Vorgänger an. Dazu gab er Donato Bramante den Auftrag zur Verbreiterung und Begradigung der Via della Lungara und zum Bau der Via Giulia, einer Parallelstraße auf der anderen Seite des Tibers. Mit der Errichtung neuer Straßenanlagen wandelte Julius das von engen und unregelmäßigen Straßen geprägte Rom um. Diese Maßnahme wurde in einer Marmorinschrift in der Via de' Banchi gelobt, welche 1512 von den ÄdiIen Domenico Massimo und Geronimo Pico gestiftet wurde. ${ }^{17}$

Zum anderen hob sich Julius II. von seinen Vorgängern dahingehend ab, indem er die Kunst in einem bisher unbekannten Ausmaß und mit allen zur Verfügung stehenden Mitteln förderte und bei zeitgenössischen Meistern der Kunst - wie Bramante, Raffael und Michelangelo - monumentale, aber umsetzbare Werke in Auftrag gab. ${ }^{18}$ Diese künstlerischen Arbeiten listete Albertini in seinem Werk einzeln auf und hob sie damit besonders hervor. ${ }^{19}$ Zu den größten Unternehmungen Julius' II. zählte der Neubau der Petersbasilika. Der Entschluss dazu ist erstmals im Jahre 1505 nachweisbar. Am 18. April

\footnotetext{
14 Übersetzung nach Pastor, Geschichte der Päpste, Bd. 3, S. 952-953.

15 Francesco degli Albertini, Opusculum de mirabilibus novae et veteris urbis Romae, Rom 1510, S. 75, http://mdznbn-resolving.de/urn:nbn:de:bvb:12-bsb11211054-1, eingesehen 10.2.2021.

16 Pastor, Geschichte der Päpste, Bd. 3, S. 897-898.

17 Ludwig von Pastor, Die Stadt Rom zu Ende der Renaissance, Freiburg im Breisgau 1925, S. 26-28.

18 Pastor, Geschichte der Päpste, Bd. 3, S. 912-913.

19 Albertini, Opusculum de mirabilibus novae et veteris urbis Romae, S. 89-90.
} 
1506 fand die feierliche Grundsteinlegung von Neu-Sankt Peter statt. ${ }^{20}$ Anfangs lehnte Julius II. die Pläne Bramantes, welche die Zerstörung Alt-St. Peters vorsahen, aus Ehrfurcht vor dem Heiligen Petrus noch ab. ${ }^{21}$ Schlussendlich nahm er sie aber doch an, da sie seiner Vorstellung eines „erneuerten Petersdoms“ entsprachen.22 Neben der Baufälligkeit der konstantinischen Basilika galt Ästhetik als Hauptgrund für einen Neubau. So gab der Sekretär und Chronist von Julius II., Sigismondo de' Conti, ${ }^{23}$ an, dass die prunkvolle Renaissancebaukunst die Architektur Alt-St. Peters übertreffen sollte. ${ }^{24}$

Ein weiteres Urteil über die Bautätigkeit von Julius II. stammte von Tommaso Inghirami, der während des Pontifikats Julius' II. zum Präfekten der Vatikanischen Bibliothek ernannt wurde. In seiner Leichenrede auf Julius II., "Pro lulio II pont. max. funebris oratio”, die er im Petersdom am 21. Februar 1513 hielt, lobte er dessen Bautätigkeit mit überschwänglichen Worten:25

„Die Stadt, die er plebejisch, unscheinbar, schmutzig fand, hat er in eine reinliche, glanzvolle, des Römernamens würdige umgeschaffen. Stellt man die innerhalb von vierzig Jahren von Savonesen errichteten Bauten zusammen, so würden sie das wahre Rom bilden. Alles übrige, man verzeihe mir den Ausdruck, waren Hütten." ${ }^{26}$

Dieses Urteil kann ein Zeugnis dafür sein, dass die unter Julius II. gesetzten baulichen Maßnahmen durchwegs positiv wahrgenommen und auch bewundert wurden. Tatsächlich kümmerte sich der Papst auch um die Sicherheit Roms, indem er die Restaurierung der Stadtmauern und die Fortsetzung der Arbeiten an der Befestigung der Engelsburg in Auftrag gab. Zudem sorgte er für die Anlegung neuer sowie die Verbesserung alter Kloaken und Wasserleitungen. Er ließ unter anderem die Aqua Virgo ausbessern. ${ }^{27}$

\subsection{Papst Leo X. und das "Goldene Zeitalter"}

Nachdem Julius II. 1513 verstorben war, wurde Giovanni de' Medici zum Papst gewählt und nahm den Namen Leo X. an. ${ }^{28}$ Mit der Wahl des Sohnes von Lorenzo il Magnifico waren die Hoffnungen auf einen Übergang des Papsttums zum Mäzenatentum im

20 Pastor, Geschichte der Päpste, Bd. 3, S. 923-924.

21 Bredekamp, Sankt Peter in Rom, S. 35.

22 Christof Thoenes, Der heilige Raum der Basilika St. Peter, in: Fabbrica di San Pietro, Vatikanstadt (Hrsg.), Der Petersdom. Mosaike, Ikonographie, Raum (Monumenta Vaticana Selecta), Stuttgart 2011, S. 16-67, hier S. 18. So lautete die Umschrift auf der 1506 von Cristoforo Caradosso geprägten Gründungsmedaille von Neu St. Peter "Templi Petri Instauratio".

23 Partridge, Renaissance in Rom, S. 50.

24 Bredekamp, Sankt Peter in Rom, S. 37.

25 Stefano Benedetti, Inghirami Tommaso, in: Dizionario Biografico degli Italiani, Bd. 62, Rom 2004, http://www. treccani.it/enciclopedia/inghirami-tommaso-detto-fedra_\%28Dizionario-Biografico\%29/, eingesehen 3.8.2020.

Tommaso Inghirami, Thomae Phaedri Inghirami Volterrani Orationes duae, hrsg. von Pier Luigi Galletti, Rom 1777,

S. 82: „[...] hanc urbem ex coenosa, humili, sordida, nitidam, magnificam, splendidam, dignamque plane Romano nomine reddidit; ut si omnia aedificia a Savonensibus in hac urbe intra quadragesimum annum facta unum in locum conferantur, haec denique vera Roma; quod reliquum fuerit, absit verbo invidia, mapalia, attegiaeque merae censeantur.", Übersetzung nach Pastor, Geschichte der Päpste, Bd. 3, S. 952.

28 Pastor, Geschichte der Päpste, Bd. 4, S. 15. 
Stile der Medici groß. ${ }^{29}$ Letztendlich trieben vor allem Humanisten die Überhöhung des Mäzenatentums Leos X. voran. ${ }^{30}$ So empfand der Humanist Egidio da Viterbo die Wahl des ersten Medici-Papstes als Anbruch eines "Goldenen Zeitalters" und als Erneuerung des augusteischen Roms. Der Augustinermönch da Viterbo, der bereits unter Julius II. zum apostolischen Prediger ernannt wurde und während des Pontifikats Leos $X$. seine "Historia viginti saeculorum" schrieb, ließ die letzte seiner zwanzig Epochen der Menschheitsgeschichte im Pontifikat Leos X. enden. In dieser Zeit siedelte da Viterbo den Höhepunkt des "Goldenen Zeitalters" an, welches von den Werken Leos X. gekennzeichnet gewesen sei. ${ }^{31}$ Was die Unterstützung von Musik, Kunst und Literatur betraf, war der Papst, trotz Geldnot aufgrund schlechter Finanzwirtschaft, äußerst freigiebig. Diese verschwenderische Freigiebigkeit nahmen die Römer*innen durchwegs positiv auf. $^{32}$

Ein weiteres positives Urteil stammte von Alessandro Gabbioneta. Der Vertreter der Herzöge von Mantua bei den Päpsten in Rom schrieb in einem Brief am 14. Januar 1517, dass die Stadt täglich schöner geworden und beständig gewachsen sei. ${ }^{33}$ Leo X. hatte in den ersten Jahren seines Pontifikats die Projekte Julius'll. fortgesetzt. Er ließ die Herstellungsarbeiten an der Via Alessandrina von Giuliano da Sangallo fortführen, die Straßen im Borgo verbessern und den Platz vor der Engelsburg umgestalten. ${ }^{34} 1516$ erneuerte Leo X. die Bulle von Sixtus IV. über die Verbreiterung und Verschönerung der Straßen.. ${ }^{35}$ So entstanden etwa 1517 die Via del Babuino, die später von Paul III. weitergebaut und in Via Paolina umbenannt wurde, und die Via Ripetta (Via Leonina), die beide zur Piazza del Popolo führten. Hierbei sollte die von Raffael und Antonio da Sangallo entworfene Piazza del Popolo direkt auf den von Sangallo entworfenen Medicipalast zulaufen. ${ }^{36}$ Der Palast blieb jedoch unvollendet. ${ }^{37}$ Wie der Kunsthistoriker Hubertus Günther zeigte, sind zahlreiche Pläne zur Urbanistik Roms erhalten. Viele davon wurden jedoch nie oder nur zum Teil verwirklicht. ${ }^{38}$ Hauptgründe dafür waren der Tod des Architekten Raffaels 1520 und jener Leos X. 1521.39

29 Günther, L'urbanistica romana, S. 547

30 Pastor, Geschichte der Päpste, Bd. 4, S. 554

31 Stefania Pasti, Le fonti della Visione di Ezechiele di Raffaello. L'Historia Viginti Saeculorum di Egidio da Viterbo e il De Partu Virginis di Jacopo Sannazaro, in: Accademia Raffaello. Atti e Studi 15 (2016), Heft 1-2, S. 8-32, hier S. $18-22$.

32 Pastor, Geschichte der Päpste, Bd. 4, S. 20-21.

33 Ebd., S. 387; Abschrift des Briefes angefertigt von Ludwig von Pastor, in: Bd. 4, Abt. 2, S. 706. Das Original befindet sich im Archivio Gonzaga in Mantua.

34 Günther, L'urbanistica romana, S. 547.

35 Pastor, Geschichte der Päpste, Bd. 4, S. 387.

36 Fagiolo dell'Arco, Das römische Pontifikat, S. 23.

37 Partridge, Renaissance in Rom, S. 25.

38 Hubertus Günther, Die Straßenplanung unter den Medici-Päpsten in Rom (1513-1534), in: Jahrbuch des Zentralinstituts für Kunstgeschichte, Bd. 1, München 1985, S. 237-293, hier S. 237, https://doi.org/10.11588/ artdok.00001294, eingesehen 8.8.2020. 


\subsection{Papst Paul III.: Der römische Papst}

Mit Alessandro Farnese wurde 1534, erstmals seit mehr als hundert Jahren, wieder ein Kardinal aus Rom zum Papst gewählt.40 Sein Pontifikat wurde insbesondere von den zeitgenössischen Literaten Luca Contile und Dionigi Atanagi als ein neues "Goldenes Zeitalter" gelobt. Es sei von Ruhe, Ordnung und einer Blüte der Freien Künste sowie der Literatur gekennzeichnet gewesen. ${ }^{41}$ Aus diesem Lob geht zum einen hervor, dass Paul III. die Kunst und Wissenschaft förderte und sich in dieser Zeit viele Literaten in Rom aufhielten. ${ }^{42}$ Zum anderen erfreuten sich die Literaten über die nunmehr eingekehrte Ordnung und Ruhe, denen eine politisch instabile, unruhige Zeit vorausgegangen war. Im Kontext des Kampfes um die Vorherrschaft in Italien zwischen Frankeich und den Habsburgern hatten Söldner Karls V. zwischen 1527 und 1528 Rom geplündert („Sacco di Roma"). ${ }^{43}$ Paul III. widmete sich daher dem Wiederaufbau der Stadt. Bereits vor der Wahl Pauls III. hatte der römische Adelige Tomarozzo den Niedergang der Stadt beklagt und forderte - mit Bezug auf die große römische Antike - einen besseren Zusammenhalt unter den zerstrittenen und in ihrer Macht geschwächten römischen Adelsfamilien. ${ }^{44}$ Auch Paul III. bediente sich der antiken Symbolik, indem er Feste mit Triumphzügen, Feuerwerken, Prozessionen und Banketten organisierte, um so den Adel Roms zufriedenstellen und diesem gleichzeitig die Macht entziehen zu können. ${ }^{45}$

Die antike Symbolik spielte auch in der architektonischen Neugestaltung Roms eine bedeutende Rolle. ${ }^{46}$ Paul III. ernannte Latino Giovenale Manetti mit einem auf den 28. November 1534 datierten Breve zum Kommissar für die Altertümer. Seine Aufgabe war der Schutz der Statuen, Inschriften und Marmorsteine vor Ausbeutung und Verfall. ${ }^{47}$ Welche Bedeutung die Rezeption der Antike in der Politik Pauls III. einnahm, zeigte sich im Bau einer Triumphstraße für den Besuch Karls V., die am Palatin, Forum Romanum und antiken Triumphbögen vorbeiführte. Der Papst präsentierte Rom in seiner antiken Größe und trat dadurch dem Kaiser - nach der päpstlichen Niederlage im „Sacco di Roma" - selbstbewusst entgegen. Durch seine rege Bautätigkeit machte Paul III. Rom zu einem „Bauplatz der Erneuerung“.48 Die Stadt erfuhr einen Aufschwung, der sich im Anstieg des Wohlstands und der Bevölkerungszahl manifestierte. ${ }^{49}$ Der anstehende Besuch Karls V. im April 1536 veranlasste Paul III. zum Umbau des Kapitols. ${ }^{50}$ Zudem ließ Paul III. Altbauten verbessern und Neubauten im Vatikan errichten, die Engelsburg ausbauen und verschiedene Kirchen restaurieren. Von der zeitgenössischen Bevölkerung Roms sehr gerühmt wurde dabei die Restaurierung der später nach dem Spital Santo

40 Pastor, Geschichte der Päpste, Bd. 5, S. 20-21.

41 Rebecchini, After the Medici, S. 173.

42 Pastor, Geschichte der Päpste, Bd. 5, S. 723.

43 Silvina Paula Vidal, Una revisione delle tesi di André Chastel su alcune rappresentazioni contemporanee del Sacco di Roma (1527), in: Archivio Storico Italiano 173 (2015), Heft 2, S. 275-312, hier S. 275 https://www. jstor.org/stable/10.2307/26229867, eingesehen 26.2.2021.

45 Ebd., S. 168.

46 Ebd.

47 Pastor, Geschichte der Päpste, Bd. 5, S. 750-752.

48 Fagiolo dell'Arco, Das römische Pontifikat, S. 23.

49 Pastor, Geschichte der Päpste, Bd. 5, S. 245.

50 Ebd., S. 751-753. 
Spirito in Sassia benannten Kirche Santa Maria.51 Aufgrund der seit 1517 drohenden „Türkengefahr" beauftragte der Papst Antonio da Sangallo mit der Errichtung neuer Befestigungsanlagen. ${ }^{22}$ Die Einwohner*innen Roms profitierten auch vom Bau neuer Straßenanlagen und von Straßenregulierungen, durch die ab 1536 zahlreiche Gassen und Vorbauten entfernt wurden..$^{53}$

\section{Negative Beurteilungen der Bautätigkeit Papst Julius' II., Leos X. und Pauls III.}

\subsection{Julius II., Donato Bramante und der Neubau der Peterskirche}

Auffällig ist der Umstand, wie viel Kritik der Neubau der Peterskirche seinem Auftraggeber Julius II. und dessen Bauleiter Bramante einbrachte. Der Gelehrte Onofrio Panvinio schrieb in seinem "De rebus antiquis eccl. Basilicae S. Petri", die Zeitgenoss*innen hätten den Abbruch der alten Basilika bedauert. Vor allem das Kardinalskollegium habe sich gegen den Abriss der alten Kirche gestellt. Panvinio beschuldigte Bramante, den Papst mit seinen Plänen für den Abriss überzeugt zu haben. Zudem habe Julius II. nicht auf die vielen kritischen Stimmen im Kardinalskollegium gehört. ${ }^{54}$ Dabei muss darauf geachtet werden, dass Panvinio seine eigene kritische Haltung durch die Stimmen der Zeitgenoss*innen zum Ausdruck bringen wollte. ${ }^{55}$ Aus welchen Gründen stieß der Neubau der Petersbasilika auf solche Ablehnung? Der päpstliche Zeremonienmeister Paris de' Grassi lieferte mit einer auf Juni 1511 datierten Aussage einen Hinweis zur Debatte: Ihm zufolge wurde Bramante - vielsagend "ruinante“ genannt - die schonungslose Zerstörung alter Denkmäler vorgeworfen. ${ }^{56}$ In der Tat wurden in vielen zeitgenössischen Quellen die Zerstörung der alten Basilika und der achtlose Umgang mit Altertümern beklagt. ${ }^{57}$ Auch Raffael vertrat diesen Standpunkt. Michelangelo beschwerte sich über den schonungslosen Abriss der Säulen der alten Konstantinbasilika. ${ }^{58}$

Als Papst Julius II. im Jahr 1513 starb, war die Peterskirche Ruine und Baustelle zugleich.59 Der Unmut über den Abriss Alt-St. Peters dauerte nach dem Tode Julius' II. an und kam auch in der zeitgenössischen Satire zum Ausdruck. Ein Beispiel hierfür ist der 1517 erschienene satirische Dialog "Simia"60 ("Affe") zwischen Bramante, dem Heiligen Petrus und dem Bologneser Alessandro Zambeccaro vor den Toren des Paradieses..$^{61}$ Verfasser war der Humanist Andrea Guarna aus Salerno, der vermutlich zwischen 1510 und 1511

51 Pastor, Geschichte der Päpste, Bd. 5, S. 756-764.

52 Ebd., S. 744-745.

53 Pastor, Geschichte der Päpste, Bd. 5, S. 245, S. 751-753.

54 Onofrio Panvinio, De rebus antiquis memorabilibus et praestantia basilicae sancti Petri apostolorum principis Vaticanae, Rom 1568 (unveröffentlichtes Manuskript), S. 372-373, https://digi.vatlib.it/mss/detail/238490, eingesehen 24.2.2021.

55 Bredekamp, Sankt Peter in Rom, S. 50-51.

56 Luigi Frati, Le due spedizioni militari di Giulio II. Tratte dal diario di Paride Grassi bolognese [...], Bologna 1886, S. 286-287, https://catalog.hathitrust.org/Record/100337169/Home, eingesehen 24.2.2021.

57 Ebd, S. 920-922.

58 Ebd, S. 928.

59 Thoenes, Der heilige Raum, S. 46.

60 Michael Petschenig/Franz Skutsch/JosefM. Stowasser, simia, in: Stowasser. Lateinisch-deutsches Schulwörterbuch, Wien 2015, S. 470.

61 Pastor, Geschichte der Päpste, Bd. 3, S. 921-922. 
sowie 1516 in Rom verweilte. ${ }^{62}$ Julius II. wird in dem fiktiven Gespräch durch Bramante als eigentlicher Verantwortlicher für die Zerstörung Alt-St. Peters beschuldigt, denn er habe lediglich den Abriss der alten Peterskirche finanziert, sich aber nicht um den Aufbau Neu-St. Peters gekümmert. Petrus würde Bramante den Zutritt zum Paradies erst erlauben, sobald der Neubau abgeschlossen sei. Damit äußerte der Autor des Dialogs indirekt Zweifel daran, ob der Neubau unter Leo X., dem Nachfolger Julius'II., vollendet werden könnte. ${ }^{63}$ Insgesamt kritisierte Guarna durch die vorkommenden Personen die Kurie, die sich seiner Meinung nach durch Korruption und Laster kennzeichnete. ${ }^{64}$ Wie aus diesem satirischen Beispiel hervorgeht, bestand in der zeitgenössischen Papstkritik Uneinigkeit darüber, wer für den Niederriss Alt-St. Peters verantwortlich war. Es kann jedoch davon ausgegangen werden, dass es hierfür nicht nur einen einzelnen Verantwortlichen gab. ${ }^{65}$

\subsection{LeoX. und die Altertümer}

Wie bereits im vorherigen Kapitel thematisiert, kritisierte Raffael den achtlosen Umgang mit römischen Altertümern unter Leo X. Von Bedeutung ist an dieser Stelle der Brief über die Altertümer Roms („Lettera a Leone $\left.X^{\prime \prime}\right)^{66}$, den Raffael und Baldassarre Castiglione an den Papst richteten. Wie aus dem Zitat am Beginn der vorliegenden Arbeit hervorgeht, warf Raffael den vorherigen Päpsten vor, antike Monumente, wie Tempel, Bogen und Säulen zu zerstören, um das daraus gewonnene Material für den Bau geistlicher und profaner Gebäude zu verwenden. ${ }^{67}$ Daher rief Raffael Leo X. zum Schutz antiker Denkmäler auf. Zudem erstellte er einen Stadtplan des antiken Roms. ${ }^{68}$ Durch ein Breve vom 27. August 1515 wurde Raffael daraufhin zum Oberaufseher aller in Rom ausgegrabenen Marmorstücke und Steine ernannt. Jeder Fund musste gemeldet werden, um zu entscheiden, ob das Fundstück als Baumaterial verwendet werden durfte oder nicht. Auch Leo X. nahm sich beispielsweise beim Wiederaufbau der Zona del Popolo vor, antike Denkmäler weder zu zerstören noch zu beschädigen. ${ }^{69}$ Doch bedeutet dies nicht, dass unter Leo X. keine Altertümer mehr in Mitleidenschaft gezogen wurden. Das Verständnis für den Erhalt von Antikem war - aus heutiger Perspektive - noch wenig ausgeprägt. So wurden etwa 1519 die Sarkophage im Mausoleum des Kaisers Honorius zerstört. ${ }^{70}$

62 Stefano Simoncini, Guarna, Andrea, in: Dizionario Biografico degli Italiani, Bd. 60, Rom 2003, http://www.treccani. it/enciclopedia/andrea-guarna_\%28Dizionario-Biografico\%29/, eingesehen 6.8.2020.

63 Pastor, Geschichte der Päpste, Bd. 3, S. 921-922.

64 Simoncini, Guarna, Andrea

65 Pastor, Geschichte der Päpste, Bd. 3, S. 928.

66 Francesco Paolo di Teodoro, Lettera a Leone X, S. 7.

67 Pastor, Geschichte der Päpste, Bd. 4, S. 467.

68 Ebd., S. 467-469.

69 Günther, L'urbanistica romana, S. 548.

70 Pastor, Geschichte der Päpste, Bd. 4, S. 551. 


\subsection{Paul III. und die Zerstörung von Bauwerken und Anlagen}

Paul III. bediente sich der antiken Symbolik, um seine Macht in Rom zu konsolidieren. Er präsentierte sich als Beschützer antiker römischer Relikte, indem er Manetti zum Kommissar für Altertümer ernannte. Dennoch zeigte er, wie schon seine Vorgänger, ein widersprüchliches Verhalten im Umgang mit antiken Überresten. Zeugnis dafür ist ein von Paul III. erlassenes Breve vom 22. Juli 1540. Damit erlaubte er den Deputierten der Fabbrica di S. Pietro die Suche nach Baumaterialien, wie etwa Marmor oder Travertin, in antiken Bauwerken. Bereits zuvor trugen Materialsucher solche Materialen von antiken Ruinen ab und verwendeten diese für neue Bauten. 1539 und 1540 trugen Materialsucher Rohstoffe für den Neubau von Sankt Peter im Forum Romanum zusammen. Auch für den Bau der Triumphstraße für Karl V. wurden Monumente des Forums beschädigt. ${ }^{71}$ Manetti ließ etwa zu, dass im Zuge des Straßenbaus die Fläche des Forum Romanum zwischen den Triumphbögen des Konstantins, des Septimius Severus und des Titus mit dem Bauschutt von antiken Monumenten und Wohnhäusern aufgeschüttet und geebnet wurde. ${ }^{72}$ So berichteten der französische Diplomat Guillaume du Bellay und sein Begleiter François Rabelais, die sich zwischen 1535 und 1536 in Rom aufhielten, von der Zerstörung von Monumenten, Kirchen und Häusern. ${ }^{73}$

Die Neugestaltung im Zuge des Besuchs Kaiser Karls V., deren Vorbereitungen Anfang 1536 begonnen hatten, führte jedoch nicht nur zur Beschädigung von Altertümern. ${ }^{74}$ Der Botschafter Fabrizio Pellegrino aus Mantua schrieb in einem Brief an den Herzog Federico Gonzaga am 22. Januar 1536, dass die Maßnahmen vor allem die arme Bevölkerung Roms getroffen hätten, welche bereits unter den Auswirkungen des "Sacco di Roma" gelitten und nur mit Mühe für ihren Lebensunterhalt gesorgt habe. ${ }^{75} \mathrm{Am}$ 28. Januar schrieb Pellegrino, dass Paul III. durch die Straßen geritten sei und den Bau von Triumphbögen, die Zerstörung von Häusern und Mauern sowie die Ebnung und Begradigung von Straßen und Gärten angeordnet habe, sodass dem Kaiser die (antike) Größe der Stadt präsentiert werden konnte. Ihm zufolge bedeutete die Zerstörung einen großen Schaden für die Stadt und ihre Vergangenheit. Mit den Worten „ma bisogna bere a questo calice" wies Pellegrino darauf hin, dass die Römer*innen die Maßnahmen hinnehmen mussten. ${ }^{76}$

\section{4. $\quad$ Fazit}

Anhand der zeitgenössischen Berichte von Botschaftern, Klerikern, Humanisten und Literaten kann die eingangs aufgestellte These nur teilweise bestätigt werden. Zum einen wurden die Maßnahmen der Neugestaltung in Quellen negativ beurteilt, da diese zur Zerstörung der Altertümer Roms zu Gunsten prachtvoller Bauten führten. So wurde

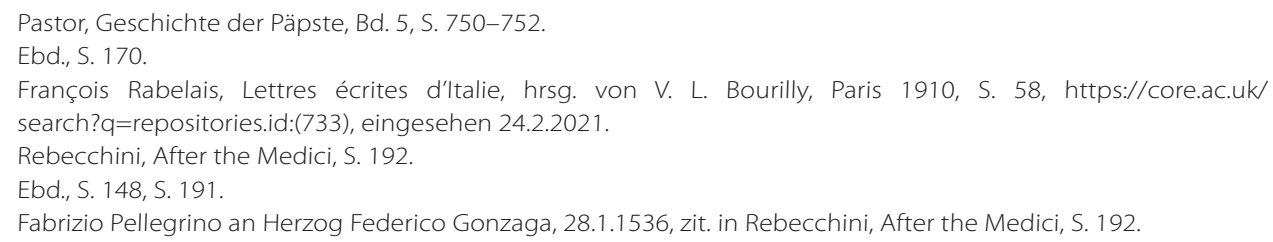


etwa die Zerstörung Alt-St. Peters unter Julius II. heftig kritisiert. Diese negativen Beurteilungen gingen einerseits auf klerikale Kreise zurück, die die Zerstörung des antiken christlichen Heiligtums nicht hinnehmen wollten. Andererseits stammte diese Kritik aber auch von Humanisten, die sich zunehmend für den Schutz der römischen Altertümer einsetzten. Bestrebungen, die Altertümer zu schützen, und die Missbilligung der Zerstörung selbiger zeigten sich auch während der Pontifikate Leos X. und Pauls III. Fabrizio Pellegrino führte in seinem Bericht vor Augen, dass die Maßnahmen zur Neugestaltung Roms unter Papst Paul III. vor allem die arme Stadtbevölkerung belasteten. Daraus kann geschlossen werden, dass die städtebaulichen Unternehmungen von Betroffenen höchst wahrscheinlich negativ wahrgenommen wurden.

Demgegenüber erfuhren die städtebaulichen Maßnahmen zur Verschönerung und Verbesserung der Straßen sowohl unter Julius II. als auch unter Leo X. und Paul III. besonderen Zuspruch. Zudem fanden bauliche Unternehmungen im Namen von Sicherheit und Gesundheit unter Julius II. positive Resonanz. Die Bautätigkeit Julius' II. wurde aufgrund der monumentalen Auftäge und des Engagements bekannter Architekten besonders gelobt. Insgesamt stellte sich heraus, dass das Lob vor allem von Seiten der Kleriker, Humanisten und Literaten stammte, welche von der Bautätigkeit der drei genannten Päpste profitierten.

\section{Literatur und Quellen}

Albertini, Francesco degli, Opusculum de mirabilibus novae et veteris urbis Romae, Rom 1510, http://mdz-nbn-resolving.de/urn:nbn:de:bvb:12-bsb11211054-1, eingesehen 10.2.2021.

Benedetti, Stefano, Inghirami Tommaso, in: Dizionario Biografico degli Italiani, Bd. 62, Rom 2004, http://www.treccani.it/enciclopedia/inghirami-tommaso-dettofedra_\%28Dizionario-Biografico\%29/, eingesehen 3.8.2020.

Bering, Kunibert, Die Peterskirche in Rom. Architektur und Baupropaganda (Architektur der Welt), Weimar 2003.

Bredekamp, Horst, Sankt Peter in Rom und das Prinzip der produktiven Zerstörung. Bau und Abbau von Bramante bis Bernini, Berlin 2000.

Fagiolo dell'Arco, Marcello, Das römische Pontifikat. Die Geschichte einer Ideologie im Spiegel einer heiligen Stadt, in: Maurizio Fagiolo dell'Arco (Hrsg.), Petersdom und Vatikan. Eine Dokumentation über das Zusammenwirken von Päpsten, Malern und Bildhauern, die Petersdom und Vatikan zu einem Wahrzeichen der Christenheit und Denkmal der Kunst machten, Freiburg im Breisgau 1983, S. 12-27.

Frati, Luigi, Le due spedizioni militari di Giulio II. Tratte dal diario di Paride Grassi bolognese [...], Bologna 1886, https://catalog.hathitrust.org/Record/100337169/Home, eingesehen 24.2.2021. 
Günther, Hubertus, Die Straßenplanung unter den Medici-Päpsten in Rom (1513-1534), in: Jahrbuch des Zentralinstituts für Kunstgeschichte, Bd. 1, München 1985, S. 237-293, https://doi.org/10.11588/artdok.00001294, eingesehen 8.8.2020.

Ders., L'urbanistica romana sotto il pontificato dei Medici, in: Henry A. Millon (Hrsg.), Rinascimento da Brunelleschi a Michelangelo. La rappresentazione dell'architettura, Mailand 1994, S. 546-551, https://doi.org/10.11588/artdok.00001273, eingesehen 8.8.2020.

Inghirami, Tommaso, Thomae Phaedri Inghirami Volterrani Orationes duae, hrsg. von Pier Luigi Galletti, Rom 1777.

Panvinio, Onofrio, De rebus antiquis memorabilibus et praestantia basilicae sancti Petri apostolorum principis Vaticanae, Rom 1568 (unveröffentlichtes Manuskript), https:// digi.vatlib.it/mss/detail/238490, eingesehen 24.2.2021.

Partridge, Loren, Renaissance in Rom. Die Kunst der Päpste und Kardinäle, Köln 1996.

Pasti, Stefania, Le fonti della Visione di Ezechiele di Raffaello. L' Historia Viginti Saeculorum di Egidio da Viterbo e il De Partu Virginis di Jacopo Sannazaro, in: Accademia Raffaello. Atti e Studi 15 (2016), Heft 1-2, S. 8-32.

Pastor, Ludwig von, Die Stadt Rom zu Ende der Renaissance, Freiburg im Breisgau 1925.

Ders., Geschichte der Päpste seit dem Ausgang des Mittelalters, Bd. 3: Geschichte der Päpste im Zeitalter der Renaissance von der Wahl Innozenz' VIII. bis zum Tode Julius' II. 1484-1513, Abt. 2: Pius III. und Julius II., Freiburg im Breisgau 1956.

Ders., Geschichte der Päpste seit dem Ausgang des Mittelalters, Bd. 4: Geschichte der Päpste im Zeitalter der Renaissance und der Glaubensspaltung von der Wahl Leos X. bis zum Tode Klemens'VII. (1513-1534), Abt. 1: Leo X., Freiburg im Breisgau 1956.

Ders., Geschichte der Päpste seit dem Ausgang des Mittelalters, Bd. 5: Geschichte Papst Pauls III., Freiburg im Breisgau 1956.

Petschenig, Michael/Skutsch, Franz/Stowasser, Josef M. simia, in: Stowasser, Lateinischdeutsches Schulwörterbuch, Wien 2015, S. 470.

Rabelais, François, Lettres écrites d'Italie, hrsg. von V. L. Bourilly, Paris 1910, https://core. ac.uk/search?q=repositories.id:(733), eingesehen 24.2.2021.

Rebecchini, Guido, After the Medici. The New Rome of Pope Paul III., in: I Tatti Studies in the Italian Renaissance 11 (2007), Heft 1, S. 147-200, https://www.jstor.org/ stable/20111825, eingesehen 8.8.2020.

Reumont, Alfred von, Geschichte der Stadt Rom, Bd. 3, Abt. 2, Berlin 1870.

Simoncini, Stefano, Guarna, Andrea, in: Dizionario Biografico degli Italiani, Bd. 60, Rom 2003, http://www.treccani.it/enciclopedia/andrea-guarna_\%28Dizionario-Biografico\%29/, eingesehen 6.8.2020. 
Teodoro, Francesco Paolo di, Lettera a Leone X di Raffaello e Baldassarre Castiglione (Biblioteca dell'“Archivum Romanicum"), Florenz 2020.

Thoenes, Christof, Der heilige Raum der Basilika St. Peter, in: Fabbrica di San Pietro, Vatikanstadt (Hrsg.), Der Petersdom. Mosaike, Ikonographie, Raum (Monumenta Vaticana Selecta), Stuttgart 2011, S. 16-67.

Vidal, Silvina Paula, Una revisione delle tesi di André Chastel su alcune rappresentazioni contemporanee del Sacco di Roma (1527), in: Archivio Storico Italiano 173 (2015), Heft 2, S. 275-312, https://www.jstor.org/stable/10.2307/26229867, eingesehen 26.2.2021.

Thekla Kollmann ist Studentin der Geschichte im 4. Semester an der Universität Innsbruck.Thekla.Kollmann@student.uibk.ac.at

\section{Zitation dieses Beitrages}

Thekla Kollmann, Die Bautätigkeit der Päpste Julius II., Leo X. und Paul III. in Rom und deren Wahrnehmung in der ersten Hälfte des 16. Jahrhunderts, in: historia.scribere 13 (2021), S. 275-286, [http://historia.scribere.at], eingesehen 22.6.2021 (=aktuelles Datum).

Creative Commons Licences 3.0 Österreich unter Wahrung der Urheberrechte der Autorlnnen. 